Sādhanā, Vol. 18, Part 1, March 1993, pp. 125-141. (C) Printed in India.

\title{
The NAL-ISRO acoustic test facility
}

\author{
S BALAKRISHNA, RANJAN MOODITHAYA and \\ S NAGABHUSHANA
}

National Aeronautical Laboratory, Bangalore 560017, India

MS received 19 July 1991; revised 28 November 1991

\begin{abstract}
The Indian Space Programme has the goals of launching a number of communication and remote sensing satellites for various uses. The acoustic exposure testing of such space bound components and systems on ground is mandatory to check their ability to withstand the extreme noise fields encountered during the trans-atmospheric flight to their final destinations in space. This paper outlines the nature and origin of this acoustic field, need for acoustic testing, establishment of a versatile groundbased high intensity acoustic-testing facility and its performance evaluation. The facility was set up as a joint project between the National Aeronautical Laboratory (NAL) and the Indian Space Research Organisation (ISRO).
\end{abstract}

Keywords. Acoustic spectra; electro-pneumatic transducers; sound pressure levels; acoustic testing; spectral control; reverberation chamber; eigen tones.

\section{Introduction}

In the entire history of human evolution, man's ability to understand and exploit the earth's environment has grown exponentially only during the twentieth century. This phenomenal growth is attributable to the integration knowledge of that has occurred during the past few centuries in physical sciences and the very versatile engineering applications that have come about synergistically during the world wars of this century and the beginnings of space programmes by various countries. Development of transistors in the late 1940's and subsequent advances in electronic devices for communication/signal processing in terms of frequency response, power handling capability, miniaturisation, packing density, thermal dissipation, high speed computation, magnetic information storage, and development of concepts of software, gradually tending towards expert knowledge and artificial intelligence, have been astounding. The human ability to communicate over large distances through electrical means, either in air or through cables, though barely a century old, has created a major revolution and is affecting all people on earth. The quantum of information that is carried through carrier frequency-based concepts has enormously increased with use of electromagnetic waves in gigahertz ranges in atmosphere or visible spectra electromagnetic waves in optical fibres on the earth's surface. This allows simultaneous 
use of many thousands of channels of information. Electromagnetic waves of such high frequencies are efficiently carried in air/space only when used in line-of-sight mode. Since the earth is spherical, its curvature has imposed many restrictions on ground-based radio communication systems. A natural solution to this curvature problem is the concept of space-based communication satellites located at large distances from earth. The satellite-based transponders overcome the earth's curvature problem and can cover a full hemisphere at large distances. Three such satellites, in principle, can accommodate communication to all points on earth. Satellite-based communication has become an engineering reality due to advances in rocket propulsion systems, computers and telemetry, culminating in synchronous satellites, which are fixed with reference to the earth. The INSAT series of satellites of India belong to this category and have made a major impact on communication in India. These satellites can also serve as weather-observation and earth resource-survey platforms by utilising pictures of the earth's surface in selected electromagnetic spectra.

To take any mass out of the earth's field of gravity so that it may become an artificial satellite, it is necessary to accelerate the object from $0 \mathrm{~km} / \mathrm{s}$ to $11-12 \mathrm{~km} / \mathrm{s}$ (escape velocity), even when the earth's rotational component is utilised by launching from the earth's equator. With contemporary technology, to carry a unit mass of operational payload to low earth orbit requires many orders of magnitude larger take-off mass of launch vehicle. Since total cost is related to the take-off mass, every effort is made to reduce it. In space, there are practically no mechanical loads acting in the microgravity situation which are comparable to the self-weight of the device on earth. Thus, in principle, the payload needs to be mechanically very light, with thermal and other considerations dictating the mechanical design.

However, while taking a payload from $0 \mathrm{~km} / \mathrm{s}$ on earth's surface to nearly $12 \mathrm{~km} / \mathrm{s}$ near orbit, a complex set of rockets is used while the vehicle accelerates through the atmosphere of nearly $100 \mathrm{~km}$ thickness. This trans-atmospheric transition time, typically about a few minutes, is a very critical stage in the life of the payload due to the energy that is being dissipated to reach the escape velocity. Due to the proximity of this energy field, the payload is exposed to many mechanical and structural loads of various frequencies, which become critical factors in its mechanical design. The vibration field due to the rocket plume and its noise is one set of mechanical loading factors. The acoustic field generated by the air-flow on the launch vehicle and the nose is the cause of another mechanical loading. The acoustic field is created by the boundary layer on separation and increases with velocity. It is likely to have bimodal peaks at transonic Mach numbers where the dynamic pressure peaks. The frequency spectrum of this noise is time-varying with spatial and temporal components. This acoustic field can damage the delicate payload which has been designed with low weight considerations. The acoustic field exposure exists only as long as the payload is in the earth's atmosphere. It is therefore necessary to qualify all payload packages for such launch exposure energy fields to assess their mechanical integrity. This paper is concerned with a ground test facility capable of addressing one such energy field exposure problem, namely acoustic field exposure studies on space-bound systems.

\section{Acoustic field exposure of space-bound packages}

The acoustic energy generated around a launch vehicle moving towards the earth's orbit is a function of the rocket thrust and the aerodynamic pressure encountered 
by the vehicle. The dynamic pressure is a function of the local atmospheric pressure, temperature and vehicle velocity. The dynamic pressure peaks at a certain altitude and thereafter its effect decreases as the vehicle moves towards the vacuum of space. Since the maximum loading encountered occurs at maximum dynamic pressure, it is informative to assess the likely altitude at which this can occur. The earth's atmosphere can be modelled for its density as follows.

$$
\ln \rho=0 \cdot 36156-1.4168 h \times 10^{-4}
$$

where $\rho=$ density, $\mathrm{kg} / \mathrm{m}^{3}, \quad h=$ altitude, $m$.

This model is based on the report by Ananthasayanam \& Narasimha (1984). The information obtained can be utilised to assess the growth of dynamic pressure on a body that is being accelerated to escape velocity.

Consider the launching of a payload at an ideal constant acceleration, so that the escape velocity of about $12 \mathrm{~km} / \mathrm{s}$ is reached in finite time. If the vehicle velocity in the atmosphere is programmed to be directly related to the altitude, i.e., $v \propto h$, then the dynamic pressure encountered can be expressed as,

$$
q=\frac{1}{2} \rho v^{2} \propto h^{2} \exp \left[0.36156-1.4168 h \times 10^{-4}\right]
$$

This expression can be differentiated with respect to altitude to obtain the maximum dynamic pressure

$$
\begin{aligned}
\partial q / \partial h & =\left(2 h-1.4168 h^{2} \times 10^{-4}\right) \exp \left[0.36156-1 \cdot 4168 h \times 10^{-4}\right]=0, \\
h & =14.116 \mathrm{~km} .
\end{aligned}
$$

Hence, the dynamic pressure loading maximum occurs at about $14 \mathrm{~km}$ altitude and, at this point, the vehicle elastic loading induced vibrations and aerodynamically induced noise can be expected to peak. If the strategy of vehicle acceleration is different, the dynamic pressure maximum occurs at a different altitude not far from the $14 \mathrm{~km}$ level. With a uniform acceleration of $20 \mathrm{~m} / \mathrm{s}$, the dynamic pressure maximum is likely to occur in a $30-50 \mathrm{~s}$ time frame after launch.

The vibration field caused by rocket-induced energy and the aerodynamic energy are mechanically coupled to the payload through various resonances of the multiple degrees of freedom launch vehicle structure. In contrast to the acoustic excitation caused by the rocket, the aerodynamic energy is coupled to the payload through the air surrounding it and is transmitted as a sound field from various noise sources. Thus, vibration response of the payload is distinctly physically different from acoustic excitation. The acoustic excitation is a hydrostatic-like coupling and cannot occur in vacuum whereas vibration excitation can. This paper is concerned with the acoustic-coupling aspects of launch vehicles and their sensitive and delicate payloads. The energy involved in acoustic coupling is dominant in the $12 \mathrm{~Hz}$ to $10 \mathrm{kHz}$ range. The lower part of the spectrum is because of the rocket during the initial launch period and the higher frequencies are due to the aerodynamic noise. This spectrum is time-varying in nature and peak acoustic excitation can occur during any phase of trans-atmospheric flight. Any ground simulation of this acoustic exposure must simulate the maximum of each component of the concerned frequency in the entire launch history and such a spectrum can be called the spectral signature of the launch vehicle. The signature is unique for each of the contemporary launch vehicles. 


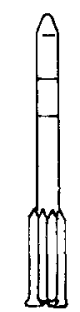

Delta

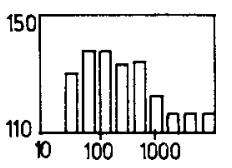

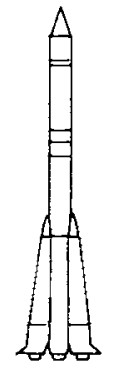

Vostok

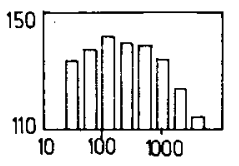

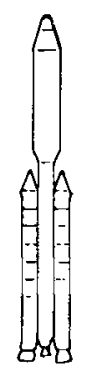

Titan $3 C$

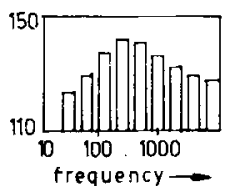

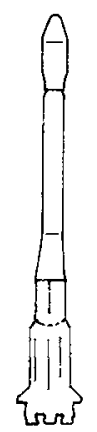

Ariane

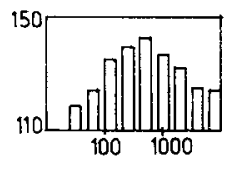

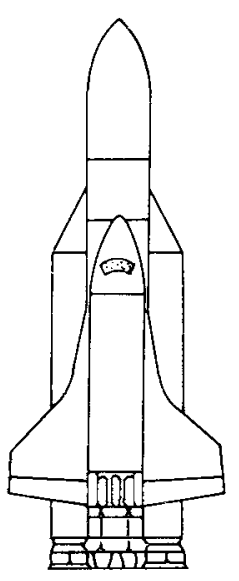

Space shuttle

Figure 1. Acoustic spectra of some launch vehicles.

\subsection{Spectral signature}

The peak spectral signatures from a few contemporary launch vehicles are presented in figure 1 . The vehicles considered are Delta, Vostok, Titan III, Ariane and NASA STS. The frequency excitation peaks that can occur during a launch and transit through the atmosphere are typically segmented in octave band ranges of $31 \cdot 5,63,125,250$, $500,1000,2000,4000$ and $8000 \mathrm{~Hz}$. The noise level is presented in decibels referred to a reference noise level of $20 \mu$ pascals. The integrated (over the frequency range) noise level is also shown as overall sound pressure level (OASPL). A study of figure 1 clearly shows that the spectral signature of each vehicle is different. The energy content in the acoustic field can be related to the integral of the signature plots over the frequency range. The acoustic energy increases with vehicle size. Further, the high frequency content also appears to increase with vehicle size. Any ground testing of space-bound packages must therefore be conducted for the signature spectra of the vehicle which will carry the payload to its final destination (NASA 1977).

\section{Acoustic testing}

India has embarked upon a programme of civilian space-bound activities aimed towards indigenous capability in design of satellites and launch vehicles, and at locating these satellites in space so as to serve for communication, land resource survey, meteorology and scientific programmes. As part of this programme, the Department of Space has been establishing the necessary infrastructure to realise these goals over the past 30 years. Amongst many sophisticated ground test facilities, the Acoustic Test Facility, set up at the National Aeronautical Laboratory (NAL) in 
1986 , is the one that deals with problems of likely damage due to acoustic energy exposure in the launch and trans-atmospheric flight phases.

Typically, an acoustic testing facility requires a large reverberation chamber, in which desired signature spectra are generated so as to expose them to space-bound packages, and an energy source to create the desired noise. A $1100 \mathrm{~m}^{3}$ reverberation chamber was considered desirable for the Indian Space Programme with about 80-100 acoustic kilowatts of energy dissipation to realise the signature spectra so as to meet the MIL-STD-810C requirements. The contemporary and most efficient method of generating large acoustic noise fields is through the use of electro-pneumatic transducers. These devices operate using air energy and have a typical 6-7\% efficiency in converting pneumatic energy to acoustic energy. Thus to generate $80-100 \mathrm{~kW}$ of acoustic energy, nearly $1000-1200 \mathrm{~kW}$ of pneumatic stored energy is necessary, to provide $6-8 \mathrm{~kg} / \mathrm{s}$ mass of air at about $207 \mathrm{kPa}$ pressure supply. To create such a pressure source, electrical and compressor systems have to be installed at fairly large capital costs. The National Aeronautical Laboratory, at its Wind Tunnel Centre, has been operating a dry filtered air compressor system to operate several wind tunnels and combustion facilities since 1964 . The system has $10 \mathrm{~kg} / \mathrm{s}$ continuous mass flow and storage capability of nearly $50,000 \mathrm{~kg}$ of compressed air at $1040 \mathrm{kPa}$. A typical acoustic facility operation for about 2-3 minutes, corresponding to the typical trans-atmospheric transition of a satellite, requires about $1000 \mathrm{~kg}$ of compressed air in one operation. This is a small fraction of the daily compressed air used at the Wind Tunnel Centre. Hence, ISRO and NAL decided to set up the Acoustic Test Facility at NAL to take advantage of a proven air supply system and reduce the capital costs in establishing such a facility. A Memorandum of Understanding was signed between NAL and ISRO for a combined project team to establish the facility. Work was started in September 1983. The first acoustic test on a test specimen was conducted in February 1986 and the project was completed in the short time period of 30 months at a cost of Rs. 1.9 crores.

\section{Generation of acoustic signatures}

\subsection{Reverberation chamber}

In order to efficiently generate any desired acoustic signature, it is necessary to utilise the property of reverberation in closed chambers while making sure that the desired spectrum is realised. Every fully enclosed volume exhibits reverberation, i.e., persistence of sound. This is caused by repeated boundary reflections of sound. These multiple reflections are functions of the speed of sound and the distance which the sound traverses between reflecting surfaces. Whenever a sound wave strikes a wall, a part of its energy is absorbed by the wall and the rest is reflected. The reflected wave in turn is partially absorbed and is partially reflected in its next encounter with a hard surface. This process is repeated till all the energy is fully absorbed by the walls. In an ideal reverberation chamber, the wall absorption is intentionally minimised by making the chamber surface nonporous and very hard, so that most of the incident energy is reflected back to the chamber. This is in contrast to anechoic chambers wherein the walls are made to absorb ideally all the incident energy. This reverberation can be formally quantified by the definition - Reverberation time is the time required for the original sound level to attenuate by $60 \mathrm{~dB}$. This is one of the key 


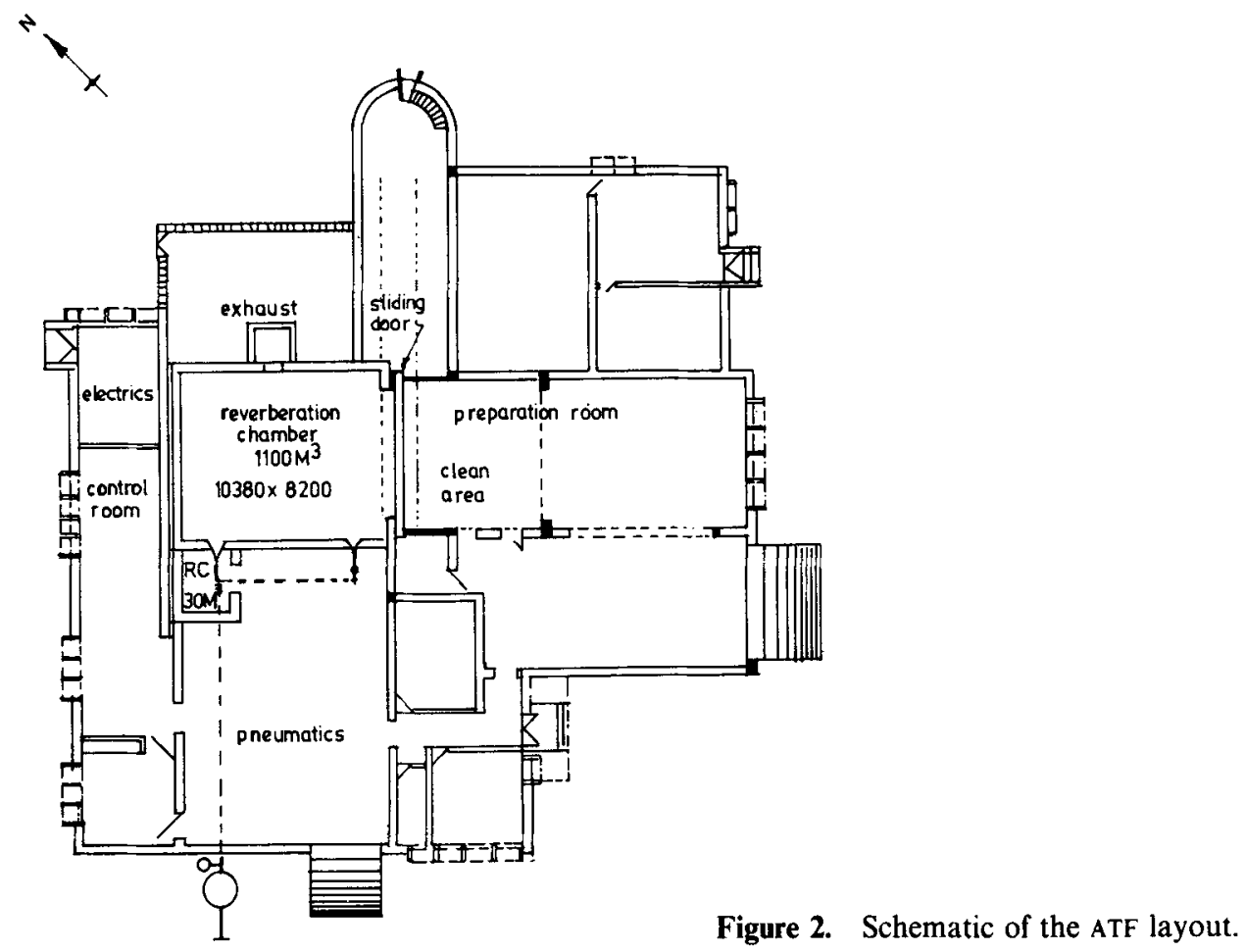

parameters deciding the chamber performance and can be expressed as (NASA 1977),

where

$$
T_{r}=60 v /[1 \cdot 085 c(a \alpha+4 m v)],
$$

$$
\begin{aligned}
c & =\text { velocity of sound, } \mathrm{m} / \mathrm{s} ; \\
a & =\text { internal surface area, } \mathrm{m}^{2} ; \\
\alpha & =\text { wall absorption coefficient; } \\
m & =\text { air absorption coefficient, } \mathrm{m}^{-1} ; \\
v & =\text { volume of chamber, } \mathrm{m}^{3} .
\end{aligned}
$$

The second important property of a reverberation chamber is the modal distribution, i.e. the frequency response of the chamber. This modal character of the chamber is a function of the dimensions of the chamber. In order to make sure that these modal frequencies are distributed uniformly so as to resemble white noise, the shape and dimensions of the chamber must be chosen properly. Typical chamber shapes that are used include rectangular (an easy to construct shape), pentagonal, and truncated pentagonal (derived from rectangular) designs and each has its advantages and disadvantages. The dimensions of the chamber should be chosen on the basis of irrational ratio of distances amongst the various internal surfaces. Being irrational, the modal harmonics of one will not coincide with the harmonics of other dimensions. Sepmeyer's cube-root-two law is one such irrational ratio-based algorithm for the design of a rectangular chamber of height $L_{z}$, breadth $L_{y}$ and length $L_{x}$. 
This law is,

$$
L_{z} / L_{y}=L_{y} / L_{x}=(2)^{1 / 3}=1 \cdot 25994 \text { or } L_{z}: L_{y}: L_{x}:: 1: 0 \cdot 82: 0 \cdot 63
$$

Thus, when a reverberation chamber of proper irrational dimensions, possessing low damping (high reverberation time), is excited by random noise, only select frequencies reverberate and others are damped. For good reverberation chamber performance, in addition to large $T_{r}$, the modal density, defined as number of frequency modes (eigen tones) per hertz (and uniform separation of the nodes), is important. This should be particularly true at low frequencies where an adequate number of eigen modes must populate the chamber. The eigen mode density of the chamber is theoretically evaluated using the expressions below.

$$
\begin{aligned}
& M(f)=D(f) n(f) \\
& M(f)=\text { modal overlap index; } \\
& D(f)=2 \cdot 2 / T_{r}=3 \mathrm{~dB} \text { bandwidth of modal resonance; } \\
& n(f)=\text { average modal density. }
\end{aligned}
$$

The modal density $n(f)$ is the average number of eigen modes that exist at the centre frequency of the octave band of interest and is a function of chamber dimensions. This can be evaluated using expressions (NASA 1977),

$$
\begin{aligned}
n(f) & =\left\{4 \pi f^{2} v / c^{3}+\pi f a / 2 c^{2}+P / 8 c\right\} \\
v & =\text { volume of chamber, } m^{3} ; \\
a & =\text { surface area of chamber, } m^{2} ; \\
c & =\text { velocity of sound, } m / s ; \\
P & =4\left(L_{x}+L_{y}+L_{z}\right), m ; \\
L_{x}, L_{y} & , L_{z}=\text { length, breadth and height of chamber, respectively, } m .
\end{aligned}
$$

The modal frequencies of the rectangular chamber can be calculated by (Beranek 1949)

$$
f_{m, n, p}=(c / 2)\left\{\left(m / L_{x}\right)^{2}+\left(n / L_{y}\right)^{2}+\left(p / L_{z}\right)^{2}\right\}^{1 / 2},
$$

where $m, n, p$ are mode numbers for $x, y, z$, taking values $0,1,2, \ldots$ etc.

The spatial nonuniformity of the acoustic noise level in the reverberation chamber is determined in terms of standard deviation, $S$, of the sound pressure level and can be estimated using the expressions (NASA 1977)

where

$$
S=5 \cdot 57\left(1+0 \cdot 23 T_{r} B\right)^{-1 / 2},
$$

$B=$ equivalent bandwidth of noise, $\mathrm{Hz} ; \quad T_{r}=$ reverberation time, seconds.

\subsection{Facility requirements}

The joint requirements of testing launch vehicle components and spacecraft payloads call for establishing a reverberation chamber of adequately large volume so as to generate sound pressure levels from 31.5 to $8000 \mathrm{~Hz}$ to an OASPL of about $152 \mathrm{~dB}$. 
The spectra for testing such components is currently delineated in MIL-STD-810C.The size of the reverberation chamber was decided on the basis of the size and volume of the largest test object and the lowest eigen tone required. Though a $300 \mathrm{~m}^{3}$ facility was initially contemplated, the size requirement was gradually iterated through 500 , 800 , and finally settled at $1100 \mathrm{~m}^{3}$. The factors that decided the present volume were: (i) that acoustic efficiency of the chamber increases with volume, and (ii) the nation's future programmes may call for larger payloads and launch vehicles.

The broad initial specification aimed at was:

$\begin{array}{ll}\text { Geometry } & \text { Rectangular } \\ \text { Volume } & 1100 \mathrm{~m}^{3} \\ \text { Dimensions } & 10.33 \mathrm{~m} \times 8.2 \mathrm{~m} \times 13.2 \mathrm{~m} \\ \text { Door size } & 10 \mathrm{~m} \times 6 \mathrm{~m} \\ \text { Acoustic power } & 80,000 \text { acoustic watts } \\ \text { Overall-sound-pressure-level } & 152 \mathrm{~dB} \\ \text { Spatial distribution tolerance } & +/-1 \mathrm{~dB} \text { in central } 1 / 3 \text { volume } \\ \text { Controllable frequency range } & 31.5 \text { to } 1200 \mathrm{~Hz} \\ \text { Maximum run time } & 480 \mathrm{~s} \\ \text { Spectral shaping control } & \text { Manual }\end{array}$

\section{Engineering description of the acoustic test facility}

The Acoustic Test Facility (ATF) consists of a reverberation chamber with a large door, an air-operated acoustic excitation system, which couples to the reverberation chamber through three exponential horns, an air-supply system to feed the electropneumatic transducers to generate the acoustic excitation and an air-exhaust system for the reverberation chamber. Each of these is now described from a design engineering viewpoint (Balakrishna et al 1986).

\subsection{Acoustic drive system}

The acoustic power required to generate on OASPL of $152 \mathrm{~dB}$ in the reverberation chamber is estimated from the following relation (Beranek 1949)

where

$$
\mathrm{OAPWL}=\mathrm{OASPL}+10 \log R-6 \cdot 2
$$

$$
\begin{aligned}
\text { OAPWL } & =\text { total acoustic power required (ref. } 10^{-12} \text { watts) } \\
\text { OASPL } & =\text { overall sound pressure level in } \mathrm{dB} \text { (ref. } 20 \mu \text { pascals) } \\
R & =\text { equivalent sound absorption area, } \mathrm{m}^{2}
\end{aligned}
$$

The power estimates were made in octave bands of frequencies from 31.5 to $8000 \mathrm{~Hz}$ for the maximum envelope spectra, taking into account variation of absorption coefficient with frequency. In practice, it is necessary to add about $6 \mathrm{~dB}$ for large chambers operating at high sound intensities. The power required is also sensitive to relative humidity of the air in the chamber. This is particularly relevant to the NAL design, since NAL air supply is dried to $60 \mathrm{ppm}$. An estimate of the power required is shown in figure 3 as a function of frequency and humidity. Based on this, the estimated power required to generate $152 \mathrm{~dB}$ at $50 \%$ relative humidity is about 40 


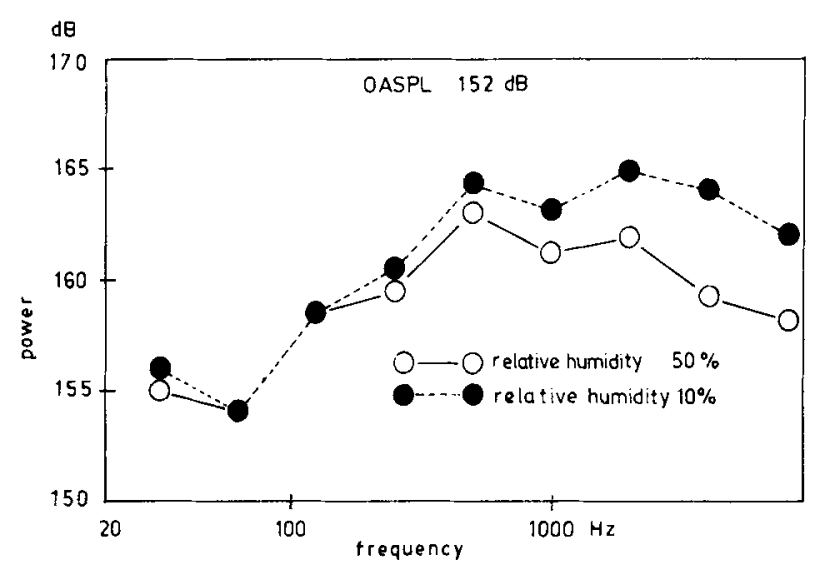

Figure 3. Power requirements at different frequencies.

acoustic kilowatts (AkW). However, in order to account for ability to control specific frequencies, particularly at low humidities, and to account for uncertainties, a power level of $80 \mathrm{~kW}$ was chosen for the NAL-ISRO chamber. There are two well-proven electro-pneumatic transducers which convert compressed air energy to sound, through an electrically modulated drive. These are the Wyle WAS 3000 and LING EPT 200. The mix of these transducers has been budgeted for $80 \mathrm{AkW}$ as two WAS 3000 's accounting for $60 \mathrm{AkW}$ and two LING EPT 200's accounting for another $20 \mathrm{AkW}$ 's. The principle of operation of the electro-pneumatic transducer is as follows. The stream of high pressure air is passed through a dynamic coil-driven cylinder with shaped slits to allow air-flow. This dynamic motion is from DC to $1200 \mathrm{~Hz}$ and can modulate the flow stream with corresponding pressure waves. An exponential horn couples these pressure waves with a good impedance match to the reverberation chamber. The two WAS 3000 s use a $25 \mathrm{~Hz}$ horn and the EPT 200 s use two $200 \mathrm{~Hz}$ horns. The wAS modulators are basically low frequency devices capable of control upto $500 \mathrm{~Hz}$, whereas the EPT 200s allow control upto $1200 \mathrm{~Hz}$. Nonlinear control at higher frequencies is possible by relying on the harmonic contents in the chamber. A view of the reverberation chamber of the NAL-ISRO ATF with an IRS satellite located at the centre is shown in figure 4 .

\subsection{Spectral control system}

The noise spectrum and the power input to the reverberation chamber is controlled by adjustment of the supply pressure to the electro-pneumatic transducers and by shaping the electrodynamic coil-current drive. A random-noise generator provides white noise and this is spectrum-shaped using $1 / 3$ octave band filters. The frequency of interest is between $20 \mathrm{~Hz}$ and $16 \mathrm{kHz}$ which corresponds to the high-fidelity stereo/audio band. Commercially available $1 / 3$ octave band filters are used to shape the drive signal. This shaped signal, after power amplification, drives the electro-pneumatic transducers. The spectrum in the ATF is the product of these audio signal filters, electro-pneumatic transducer response, and reverberation chamber response, cascaded together. After the desired spectrum shape has been established, the magnitude control is done through a simple linear attenuator. The reverberation chamber spectrum is monitored by a condenser microphone, whose output is analysed by a real-time spectrum analyser. The spectrum is observed by the operator and he manually adjusts the $1 / 3$ octave filters and signal magnitude to obtain the desired 


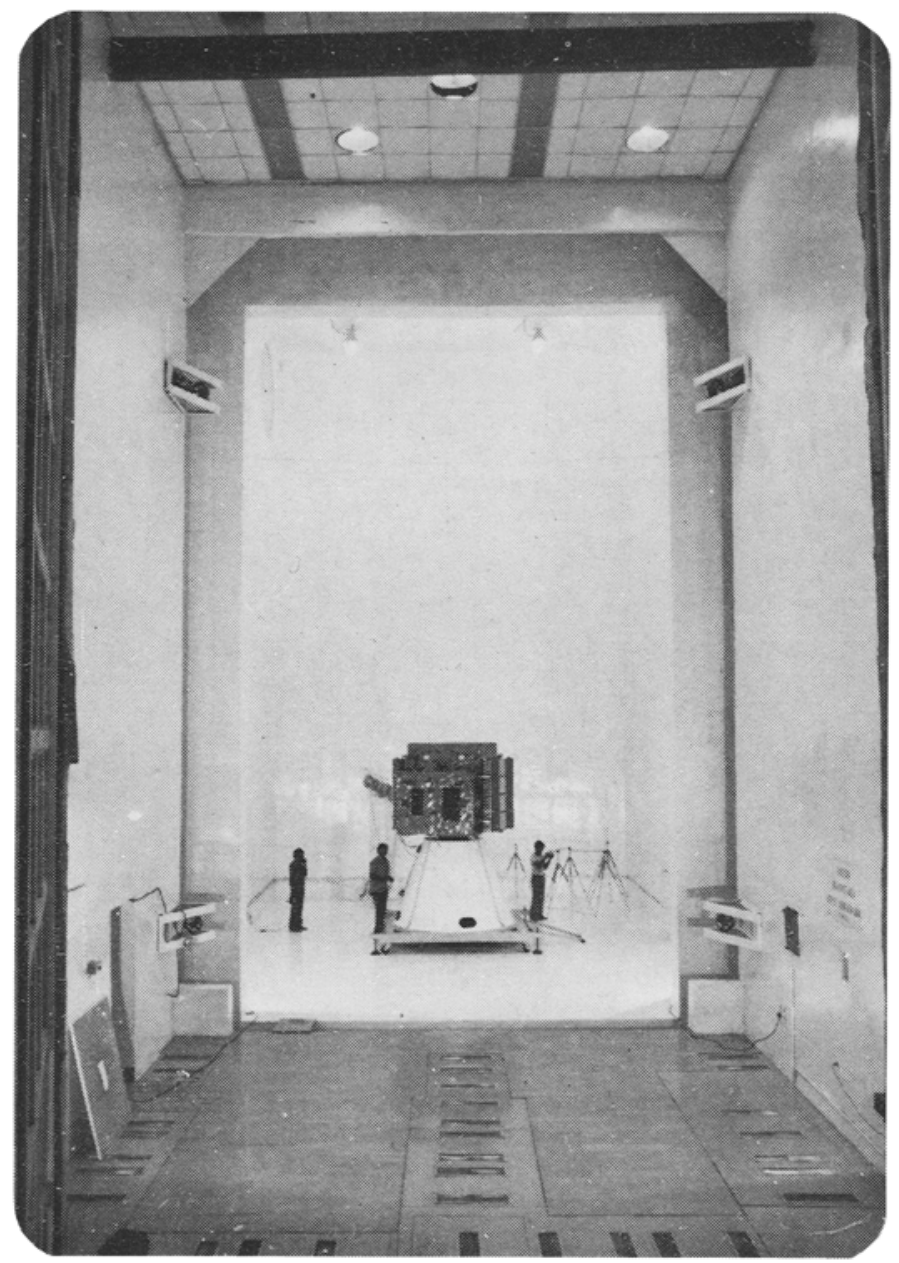

Figure 4. The IRS under test in the reverberation chamber.

spectrum in the chamber prior to a specimen test. Figure 5 shows a schematic diagram of noise generation and control.

\subsection{Air supply system}

Design of the acoustic facility is based on $80 \mathrm{AkW}$ of power; it requires clean air supply at a pressure in the range of $140-200 \mathrm{kPa}$ and a mass flow of the order of $6-8 \mathrm{~kg} / \mathrm{s}$. This mass flow is derived from the NAL central air supply system with a finite volume of $4000 \mathrm{~m}^{3}$ and a maximum pressure of $1040 \mathrm{kPa}$. The distance of the ATF from the central air supply is about $200 \mathrm{~m}$. In order to reduce the flow losses, a pipe diameter of $0.2 \mathrm{~m}$ is chosen, which drops less than $15 \mathrm{kPa}$ pressure. This supply line is terminated at a $13 \mathrm{~m}^{3}$ pressure vessel which acts like a line buffer to prevent line fluctuations.

The second issue is to reduce the supply pressure which varies from 1040 to $620 \mathrm{kPa}$, to a constant supply pressure of $160 \mathrm{kPa}$. This is realised by an electro-hydraulically driven pressure regulating system which senses the supply pressure to ATF and compares it with the set point, uses the error to adjust the throttle valve to maintain 


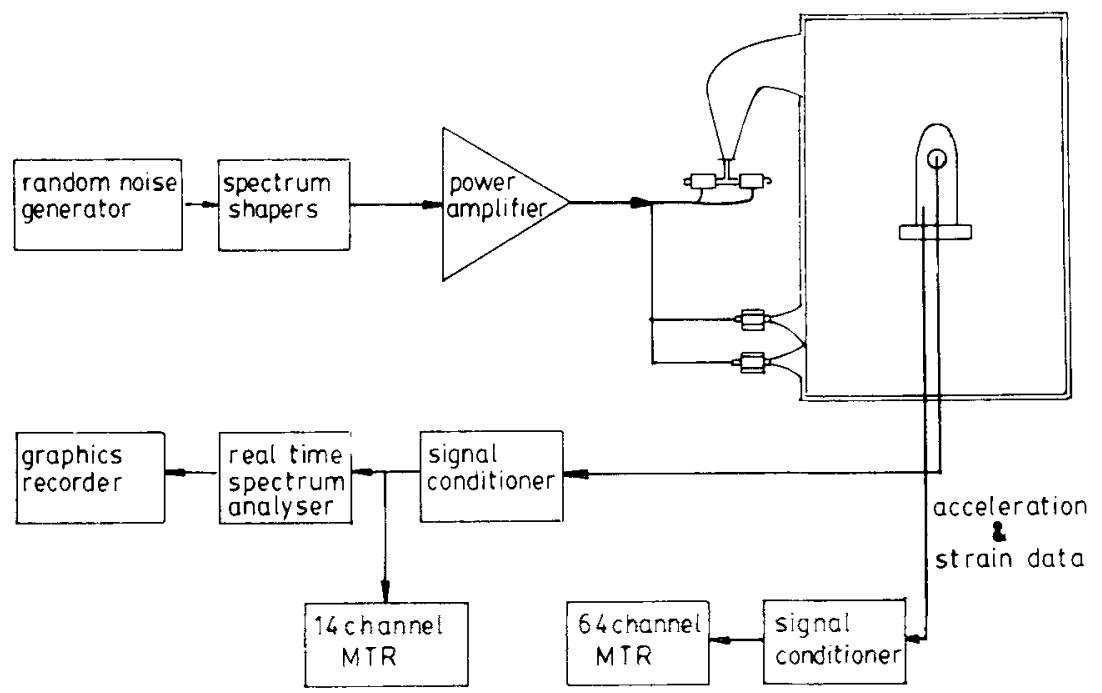

Figure 5. Schematic of the facility instrumentation.

the set pressure within $3 \mathrm{kPa}$ of the value. The NAL wind tunnels have such regulating systems capable of tight pressure control for mass flows upto $1000 \mathrm{~kg} / \mathrm{s}$. One such NAL design is used for regulating the ATF supply. The regulated flow is taken to three branches, each with a diaphragm operated air-to-open valves. Each valve is connected to a cyclonic filter which feeds to each of the four electro-pneumatic transducers. These solenoid-operated diaphragm valves serve as control elements to control the mass flow through the transducers. The electrical/pneumatic controls are terminated in the control room to provide easy supervisory control of the air-flow. The diaphragm-operated valves are fed from an instrument air-supply and are lubricated for easy and reliable operation of solenoid valves. Pressure switches on the main process and instrumentation lines provide information to the control room about the status and for safety interlocks.

The electro-pneumatic transducers, two WAS 3000s from Wyle Laboratories, consume about $170 \mathrm{~m}^{3}$ and the two high frequency Ling EPT-200s consume about $85 \mathrm{~m}^{3}$ of air. Thus a total of about $250 \mathrm{~m}^{3}$ of air is fed into the reverberation chamber, and this air leaves the reverberation chamber through a passive silencer duct into the open atmosphere. The chamber operates usually at $0.1 \mathrm{~m}$ of water pressure above atmospheric pressure. Since the NAL air supply is dried to $50 \mathrm{ppm}$ of dryness (corresponding to $223 \mathrm{~K}$ dew point), in successive operations of the facility, the chamber which starts with ambient humidity becomes very dry. This dry resident air in the chamber reduces the acoustic efficiency at higher frequencies, since air absorption is a complex function of humidity. Presently, plans are being made for humidifying the chamber continuously during facility operation with about $0.04 \mathrm{~kg} / \mathrm{s}$ of steam. Another solution is to open the facility door after every operation to let in ambient air, but this is not very effective because of the captive and air conditioned nature of the approach chamber, with limited mass turnover capability. 


\subsection{Reverberation chamber}

The dimensions of the reverberation chamber are determined mainly by the following criteria.

(a) Ability to accommodate the largest test specimen ( $\max$ specimen height $=10 \mathrm{~m}$ ).

(b) Lowest test frequency band $(31.5 \mathrm{~Hz}$ octave band).

(c) Number of eigen modes in the lowest octave band (at least one mode per hertz).

Based on the above criteria and using (5)-(7), the dimensions of the reverberation chamber were fixed at $13.2 \mathrm{~m} \times 10.3 \mathrm{~m} \times 8.2 \mathrm{~m}$.

The reverberation chamber has been made out of reinforced cement concrete. The chamber has been designed as an ideal pressure vessel, with an internal pressure of $13 \mathrm{kPa}$ to determine the reinforcement. This corresponds to about $2 \%$ of the cross-section being steel. The chamber walls are of $0.5 \mathrm{~m}$ thick concrete. The chamber stands on its own raft foundation and is isolated from the rest of the facility buildings. It has a number of penetrations, the largest being the $10 \mathrm{~m} \times 6 \mathrm{~m}$ door opening on the $13.2 \mathrm{~m} \times 8.2 \mathrm{~m}$ face of the chamber. This penetration is concrete-finished. The next biggest penetrations are for the two $25 \mathrm{~Hz}$ horn entries, which are of about $2 \mathrm{~m}$ diameter each. Each of these is made out of $0.01 \mathrm{~m}$ rolled steel sheet and has a welded flange. These penetrations, located at the top of the wall, are strongly welded to the chamber reinforcement. Presently, only one of these is in active use and the other, plugged with loose brick mortar, is for possible future use. These penetrations are located on the $13.2 \mathrm{~m} \times 10.4 \mathrm{~m}$ face, which is the wall between the pneumatic air supply bay and the chamber. The same wall, at the bottom corner, houses four $1 \mathrm{~m} \times 1 \mathrm{~m}$ square penetrations. These are also made of $0.01 \mathrm{~m}$ steel sheets with flanges and are strongly welded to the chamber reinforcement. A number of $0.2 \mathrm{~m}$ diameter pipes have been included as penetrations for viewing from the control room and to carry various services to the chamber, like electrical power, air, vacuum and signal cables. The chamber was built using a slip-form technique and uses a rich cement mix to provide high strength without surface pores. Vibrators were extensively used in this phase. The penetrations were welded before the concrete work began. The chamber was subsequently cleaned with high pressure water-jets, and epoxy-puttied before finishing with high quality epoxy paint with a high hardness finish for its internal surface. This was done with a view to increase reverberation time. The chamber has a number of high pressure mercury vapour lamp fittings of recessed shape imbedded in the ceiling. The three walls carry a number of mechanical anchors in a uniformly spaced matrix to allow mechanical anchoring or suspension of test payloads or services. The floor also carries a number of recessed anchor points, normally covered up flush, to provide strong structural anchors to test specimens. A $2 \mathrm{~m} \times 2 \mathrm{~m}$ exhaust opening has been made at bottom centre in the $13.2 \mathrm{~m} \times 10.4 \mathrm{~m}$ face opposite to the horn walls, which leads to a duct housing the silencer. The passive silencer has been designed to provide $60 \mathrm{~dB}$ noise attenuation at the mass flow normally used. The chamber door face segment of the floor has been lipped to allow movement of a wide base door structure.

\subsection{The $11 \mathrm{~m} \times 7 \mathrm{~m}$ facility door}

A major problem faced during the design of the Acoustic Test Facility was in respect of the reverberation chamber door. This door opening is nearly $60 \%$ of the size of one wall. For good noise attenuation, the door must ideally have the same type of 
construction as the chamber wall itself, pointing to the use of a very heavy concrete door. Assuming a density of 3.2 for ferro-concrete, a door of a size $11 \mathrm{~m} \times 7 \mathrm{~m} \times 0.5 \mathrm{~m}$ should weigh nearly 120 tonnes. The major issue for design was one of articulation of this door by $8 \mathrm{~m}$, and its support during door movement in closed, partially open and open conditions. For such large weights and sizes, design of a hinge, its fabrication and alignment, are very formidable. Hence, the unique concept of a free standing $11 \mathrm{~m} \times 7 \mathrm{~m} \times 0.5 \mathrm{~m}$ door (to cover the $10 \mathrm{~m} \times 6 \mathrm{~m}$ opening) mounted on a railway-type broad gauge bogie was used. In railway applications, weights of 120 tonnes are very common and bogies can easily handle such weights. Second, movement problems are trivial on a railway track. The first major issue for design of this door was thus the static balance problem. For an internal pressure of 1700 pascals (under door-closed conditions), 67.5 tonne-metre is the destabilising moment. With a half-track width of $0.75 \mathrm{~m}$ and mass of 120 tonnes, the restoring moment is 90 tonne-metre, assuring stability even at the worst internal pressure. This door can be moved across the door opening of the chamber and would serve in sealing it, provided there are no gaps between the two.

With typical engineering finish, for the NAL-ISRO ATF door, the gap has turned out to be about $0.04 \mathrm{~m}$, root mean squared (with variations of about 0.013 peak). The problem of sealing this gap whose length runs all around the door (approximately $32 \mathrm{~m}$ ) was then addressed. A special seal was developed in-house at NAL using a custom design. A vulcanised rubber base of $35 \mathrm{~m}$ length, $0.32 \mathrm{~m}$ width and $0.015 \mathrm{~m}$ thickness was made in the shape of the outline of the door with a sandwiched steel mesh. This could be mounted on the door using bolts all around. In the middle of this vulcanised base, a $0.08 \mathrm{~m}$ fire hose certified to $1350 \mathrm{kPa}$, was vulcanised for a width of $0.02 \mathrm{~m}$ of its surface. The rest of the hose was free and the tube could be pressurised to $1000 \mathrm{~Pa}$ to realise a $0.06 \mathrm{~m}$ inflatable seal of $35 \mathrm{~m}$ length. Appropriate pneumatic fittings were specially fabricated for this seal. A special vulcanising machine capable of vulcanising $1.5 \mathrm{~m}$ lengths of hose or backing was made. This machine worked at $423 \mathrm{~K}$ and used flat electrical heaters with thermostats. The four corners of $1 \mathrm{~m}$ radius had to be specially vulcanised in a separate machine. Special unvulcanised rubber rolls were used with appropriate chemicals to obtain the seal. The seal was put through peeling strength and pressure tests before being mounted on the door. While moving the door, the seal needs to be subjected to vacuum in order to collapse it so that no gouging takes place. Only after the door is in the final position covering the chamber is the seal fully inflated to cover the gaps, being fed from a regulated pressure supply of about $100 \mathrm{kPa}$. Since the seal is on the mobile door frame, flexible pneumatic hoses carry the air service to the seals. The door body also carries the vacuum pump. A changeover valve transfers the seal from the pressure to the vacuum line. The door motion logic is interlocked to the vacuum requirement on the seal. The door position microswitch signals the control room to prevent operation of the facility unless the door is fully closed. The facility can not be operated with the door partially open or without seal inflation. This seal has worked very well for the past five years except for some minor gouging damages which were easily repaired.

\subsection{Environmental control and services}

The NAL/ISRO ATF has provision for receiving sealed packages of large sizes and systematically moving them through an unloading room to a preparation room, which is air conditioned when necessary, and finally to the 100,000 class clean room 
at the entry to the reverberation chamber. This has provision for air-lock entry for personnel. The satellite is checked prior to acoustic exposure, and can be moved in to the reverberation chamber. If necessary the specimen can be covered using noise transparent tents.

The ATF facility has service rooms for the electrical power substation, air conditioning plants, and door parking annex which are appropriately located. The building also has a water supply tank to feed the cooling system for the Ling EPT transducers, which work on a passive head of water.

\subsection{Data acquisition system}

The main purpose of acoustic testing is to expose the specimen to the desired spectrum of noise, both in terms of magnitude and frequency. Thus two types of data acquisition need to be made. The first is to be able to record the exposed spectra of the noise, and is a facility safety, control and supervision requirement.

The second is specimen-oriented and hence changes from campaign to campaign. Typically, the data acquisition is for recording the following specimen responses.

- Structural vibration/acceleration.

- Strain at desired points through strain gauges with power supply and amplifier.

- Sound pressure level at desired points.

- Visual records.

The NAL-ISRO facility has 70 channels of analog magnetic instrumentation tape recorders of $8 \mathrm{kHz}$ bandwidth, and 52 charge amplifiers for piezoelectric transducers. The recorded data can be analysed on a spectrum analyser to obtain the power spectral density and amplitude information. Further, many kinds of specimen specific instrumentation are also used but are not a part of the ATF facility.

\section{Performance/characterisation of the reverberation chamber}

The performance of the reverberation chamber is quantified in two sets of calibrations. The first is the evaluation of the chamber reverberation time for various frequencies and for white noise. The second calibration, and a definitive one, is the evaluation of the spatial and temporal magnitudes of the spectra of the chamber. The results of these tests are now presented.

\subsection{Chamber reverberation time}

The chamber reverberation time has been evaluated by recording the decay of noise and evaluating the $60 \mathrm{~dB}$ loss time. For this, an internal speaker of good response is used and the reverberation time $\left(T_{r}\right)$ evaluated using white noise and pure sine wave excitation at $1 / 3$ octaval frequencies. A summary of these results is provided in figure 6 . The design goals set out initially have also been indicated. These have been generally met, but there has been a shortfall in the frequency range of 100 to $1000 \mathrm{~Hz}$. These tests were performed prior to the commissioning of the ATF. But this shortfall does not affect the facility performance as this is compensated for in the filter settings. 


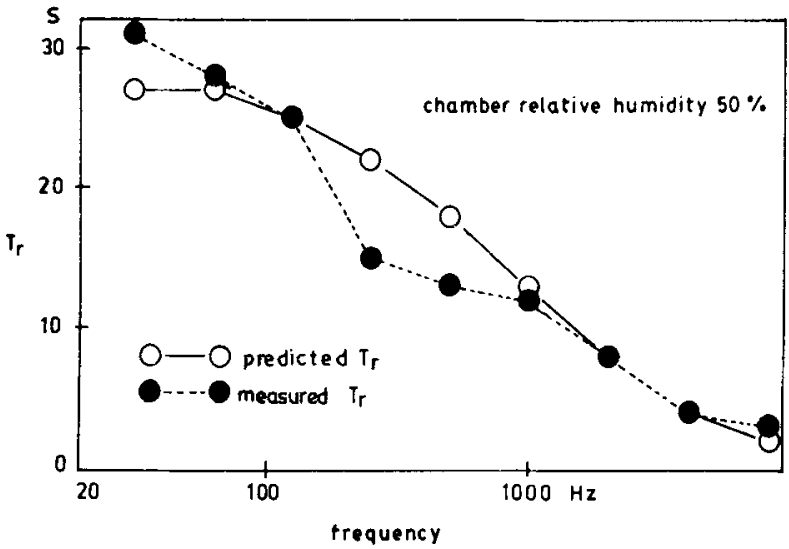

Figure 6. Reverberation time at different frequencies.

\subsection{Spatial distribution}

The chamber noise level distribution has a spatial feature which can be upset by intrusion of the test specimen. As long as the specimen size is within a few percent of the chamber volume, the empty chamber characterisation provides a good idea of the uniformity of noise distribution within the chamber. Extensive tests have been conducted to calibrate the facility for the empty chamber performance covering the full space. The tests consist of using a matrix of 48 microphones in a grid of $6 \times 8$, each spaced $1.2 \mathrm{~m}$ from one another in a rectilinear disposition. This covers an area of $6 \mathrm{~m} \times 7.2 \mathrm{~m}$ in the floor area of $8.2 \mathrm{~m} \times 10.4 \mathrm{~m}$ with only minor wall space being ignored. This mesh of 48 microphones was set up at heights of $1.2 \mathrm{~m}, 2.4 \mathrm{~m}, 3.6 \mathrm{~m}$,

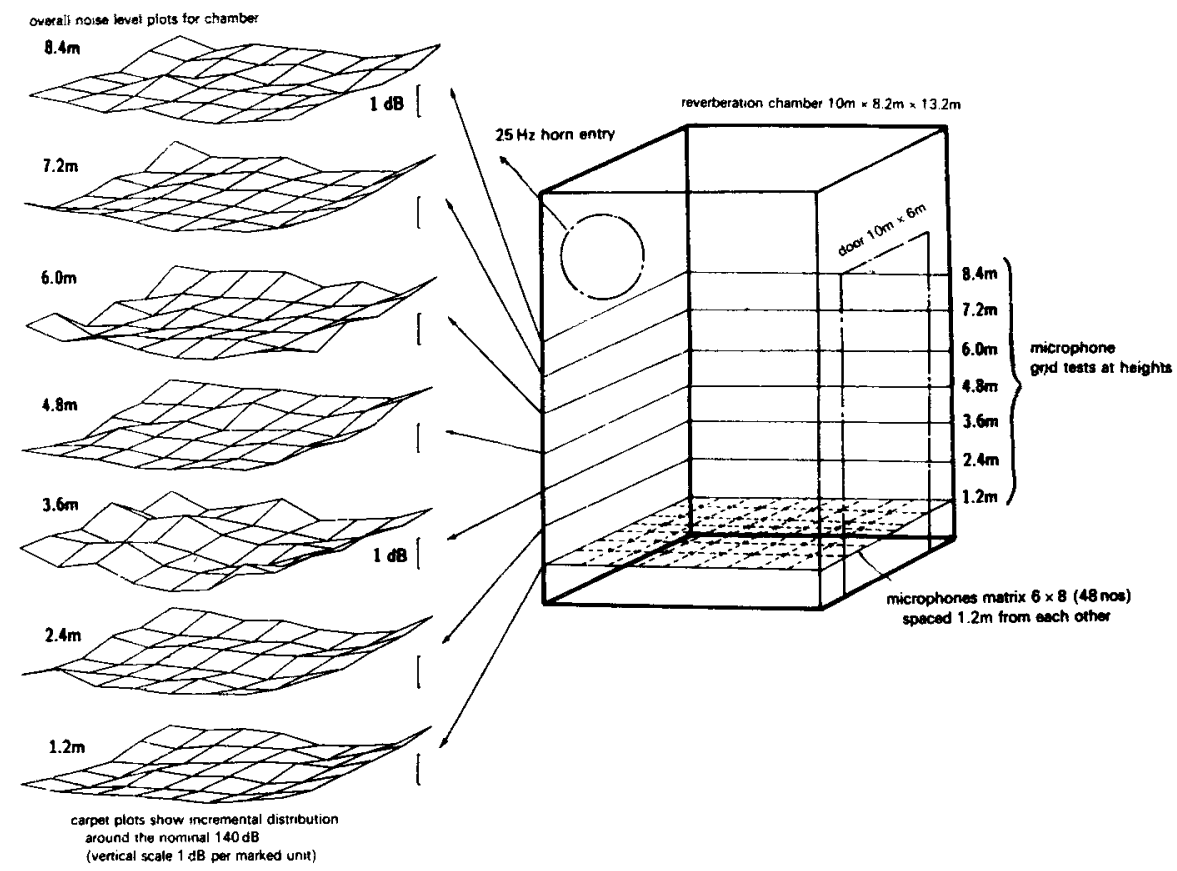

Figure 7. Spatial distribution in the chamber. 
$4.8 \mathrm{~m}, 6.0 \mathrm{~m}, 7.2 \mathrm{~m}$ and $8.4 \mathrm{~m}$ from the floor in different experiments. A nominal noise level of $140 \mathrm{~dB}$ was set and spatial distribution (Cypher et al 1974) of the chamber was recorded at the octaval band frequencies of $31 \cdot 5,63,125,250,500,1000,2000$, 4000 and $8000 \mathrm{~Hz}$. The OASPL was also recorded. Figure 7 shows typical carpet plots of the OASPL distribution in the reverberation chamber. The variation is clearly within $1 \mathrm{~dB}$ of the set value. Only the $3.6 \mathrm{~m}$ height shows the maximum irregularity. Similar plots are available for the octave band frequencies also. A second series of tests was conducted for the central $1 / 3$ volume of the chamber in a finer mesh of $0.6 \mathrm{~m}$ spacing. This distribution is also well within the $1 \mathrm{~dB}$ variation around the nominal value.

\section{Utilisation of the acoustic test facility}

The NAL-ISRO facility has been used extensively to qualify a number of Indian satellites. Acoustic testing is carried out during various stages in the development of a satellite. The first stage of testing is after a structural model has been designed and fabricated. The acoustic tests are carried out at this stage at a noise level $4 \mathrm{~dB}$ in excess of the final launch vehicle signature spectrum. These tests are likely to reveal potential loading problems and this information is used to finalise the detailed structural design of the satellite. The second stage of testing is on the engineering model and is carried out at the noise level of the final launch vehicle spectra. After successfully completing this phase, the final flight model is fabricated and is acoustically tested at the noise level and spectrum specified for the launch vehicle.

In a typical test programme, the satellite under test is transported in a vehicle fitted with special suspension fittings and is unloaded in the receiving area of the ATF. The environment is thoroughly cleaned and sealed before opening the satellite and moving it to the preparation area, where the external sensors are mounted, wired and checked using appropriate signal-conditioning equipment. The satellite is then moved on to the clean area where the satellite is prepared for final acoustic exposure. The acoustic facility is now operated empty to set the desired spectra in terms of magnitude and frequency, taking into account the likely absorption by the specimen. The controls are fully frozen at this level. The satellite is now moved into the reverberation chamber on a dolly and is located in the centre of the facility. The satellite is tented, if required, to prevent condensation on the specimen. Some supervisory microphones are located at judicious locations to monitor the health and the satellite is now ready for final tests.

During the past five years, the satellites tested in the ATF include the Indian Remote Sensing Satellite (IRS) - structural, engineering and flight models, Stretched Rohini Satellite Series (SROSS) - structural, engineering and flight model, and the Indian National Satellite (INSAT) II - structural model. In addition to these, several launch vehicle segments and solar panels have been subjected to acoustic testing for evaluation and qualification. So far nearly 880 test runs have been conducted covering nearly six families of satellites and launch vehicles. Some typical problems identified during acoustic evaluation have keen -cracking of solar panels and inadvertent heat-shield release mechanism operation.

The Acoustic Test Facility has a scheduled load for tests on all ISRO designed satellite systems/launch vehicle components till the turn of the century. The facility will also be used to conduct acoustic fatigue tests on composite structural elements that are likely to be exposed to large noise doses near the engine, like the rudder or 
other control surfaces. The facility capability may be enhanced by installation of a progressive wave tube, and a $30 \mathrm{~m}^{3}$ chamber capable of $165 \mathrm{~dB}$ OASPL test capability, if the need arises.

\section{Conclusions}

The NAL-ISRO Acoustic Test Facility is a member of the family of ground test facilities that are essential for the country to be self-sufficient in having a space programme for launching satellites that are designed, fabricated, space-located and operated indigenously. The ATF was conceptualised, designed and commissioned in a short time of less than three years.

The authors wish to acknowledge the extensive support received by the joint NAL-ISRO team from various divisions of NAL/ISRO, ATF staff and the Civil Engineering Department of ISRO in establishing the facility. They further wish to record the guidance received from Dr S R Valluri and Prof R Narasimha of NAL and Professors $S$ Dhawan and U R Rao of ISRO.

\section{References}

Ananthasayanam M R, Narasimha R 1984 A proposed international tropical reference atmosphere, Rep. 84 FM 5/84 AS4, Department of Aeronautics, Indian Institute of Science, Bangalore, May

Balakrishna S, Ganesh V S, Muralidhar S J, Nagabhushana S, Viswanathan R 1986 Acoustic test facility description manual, NAL PD SE 8619, December

Beranek L L 1949 Acoustic measurements (New York: John Wiley)

Cypher H D, Munson A N, On F J 1974 Comparative evaluation of predicted and measured performance of a $68 \mathrm{cu} \mathrm{m}$ truncated reverberant noise chamber, GSFC. NASA, May

NASA 1977 General environmental test specifications for spacecraft \& components, NAS TM-X $74375 / \mathrm{N} 77-73982$ 